\title{
Nambu representation of an extended Lorenz model with viscous heating
}

Article

Accepted Version

Creative Commons: Attribution-Noncommercial-No Derivative Works 4.0

Blender, R. and Lucarini, V. (2013) Nambu representation of an extended Lorenz model with viscous heating. Physica $D$ : Nonlinear Phenomena, 243 (1). 86 - 91. ISSN 0167-2789 Available at https://centaur.reading.ac.uk/71521/

It is advisable to refer to the publisher's version if you intend to cite from the work. See Guidance on citing.

Published version at: http://www.sciencedirect.com/science/article/pii/S0167278912002497

Publisher: Elsevier

All outputs in CentAUR are protected by Intellectual Property Rights law, including copyright law. Copyright and IPR is retained by the creators or other copyright holders. Terms and conditions for use of this material are defined in the End User Agreement.

\section{www.reading.ac.uk/centaur}

\section{CentAUR}

Central Archive at the University of Reading

Reading's research outputs online 


\title{
Nambu representation of an extended Lorenz model with viscous heating
}

\author{
R. Blender ${ }^{\mathrm{a}}, \mathrm{V}$. Lucarini $^{\mathrm{a}}$ \\ ${ }^{a}$ Meteorologisches Institut, KlimaCampus, Universität Hamburg, Grindelberg 5, 20144 \\ Hamburg
}

\begin{abstract}
We consider the Nambu and Hamiltonian representations of RayleighBénard convection with a nonlinear thermal heating effect proportional to the Eckert number (Ec). The model we use is an extension of the classical Lorenz63 model with 4 kinematic and 6 thermal degrees of freedom. The conservative parts of the dynamical equations which include all nonlinearities satisfy Liouville's theorem and permit a conserved Hamiltonian $H$ for arbitrary Ec. For $E c=0$ two independent conserved Casimir functions exist, one of these is associated with unavailable potential energy and is also present in the Lorenz-63 truncation. This Casimir $C$ is used to construct a Nambu representation of the conserved part of the dynamical system. The thermal heating effect can be represented either by a second canonical Hamiltonian or as a gradient (metric) system using the time derivative $\dot{C}$ of the Casimir. The results demonstrate the impact of viscous heating in the total energy budget and in the Lorenz energy cycle for kinetic and available potential energy.
\end{abstract}

Keywords: Rayleigh-Bénard convection, Nambu mechanics, Lorenz equations, Viscous heating

\section{Introduction}

The Nambu representation is an extension of Hamiltonian dynamics with the aim to formulate dynamical equations satisfying the Liouville theorem [20]. In state spaces with dimension above two further conserved quantities besides the Hamiltonian can be used. Thus Casimir functions can be considered as additional Hamiltonians. This concept is useful in dynamical systems when a physical interpretation of total energy is not available. Nambu systems incorporate noncanonical Hamiltonian systems with the conservation laws being Casimirs of the Poisson tensor. Algebraic properties are formulated in terms of anti-symmetric and cyclic extensions of Poisson brackets. The development of

\footnotetext{
Email addresses: richard.blender@zmaw.de (R. Blender), valerio.lucarini@zmaw.de
} (V. Lucarini) 
Hamiltonian low-order models with additional conservation laws has been also put forward in terms of gyrostat concept [5, 4]. The Nambu representations of hydrodynamics [21] has shown up to be useful in the design of numerical algorithms in geophysical fluid dynamics since they deliver a general tool to preserve conservation laws $[28,29]$. The Casimirs of ideal hydrodynamics have been explicitly derived in the noncanonical Hamiltonian representation [10]. The application of Nambu mechanics in meteorology was put forward in recent years $[23,32]$.

The continuous Rayleigh-Bénard convection can be recast in a Nambu system using total energy and a Casimir that is considered resulting from Kelvin's circulation theorem [2] or as 2D analogue of helicity [27]. Viscous dissipation is present in a symmetric bracket using the difference between total energy and unavailable potential energy. Symmetric brackets have been proposed as a natural extension of symplectic brackets and the combined system has been coined 'metriplectic' [19] (combining metric and symplectic systems).

Saltzman derived the Fourier mode truncations of the 2D Rayleigh-Bénard convection in the Boussinesq approximation [30]. The three-mode truncation of these equations in the classical Lorenz-63 model [12] includes a mode for the stream-function, a mode for temperature perturbation, and a mode for the vertical stratification. Lucarini and Fraedrich [16] included heating by viscous dissipation characterized by the Eckert number $E c$ in a low-order model without shear flow. In order to incorporate this effect, the Lorenz-63 model has to be extended to 10 degrees of freedom including four stream function, four temperature perturbation, and two vertical stratification modes.

Some of the results presented in [16] suggest that the consideration of the Ec terms is relevant. First, it was found that when $\mathrm{Ec}=0$ the system obeys a symmetry, which, by Noether's Theorem, corresponds to a conserved quantity. Such a symmetry is broken when positive values of Ec are considered, with the result that the phases of the temperature and streamfunctions are efficiently mixed and degenaracies are removed in the system, so that only one vanishing Lyapunov exponent is found. In [17], it was proposed that neglecting the heating effect due to viscous dissipation could be responsible for the observed imbalance of the global energy balance of the state-of-the-art global climate models.

The dynamical system given by the Lorenz- 63 equations can be decomposed in a conservative part which conserves volume in phase space and a divergent part related to forcing and dissipation. The conservative part has two conserved quantities, a Hamiltonian $H$ and a Casimir $C$, which can be used to determine the dynamics in a three-dimensional Nambu system $[20,22]$ so that $(\dot{X}, \dot{Y}, \dot{Z})=\nabla C \times \nabla H+\nabla \Phi$, where we have used the classical notation. Forcing and dissipation is included in the gradient of $\Phi$. Thus Nambu dynamics provides a clear geometric view of the nonlinear dynamics and yields hints to the geometry of the strange attractor in the forced and dissipative equations $[22,1]$. The Nambu structure of a six mode truncation of Saltzman's [30] convection model has been presented recently [3]. The derived model has 5 modes in common with the model studied here.

The aims of this publication are (i) to present the Nambu representation of 
the conservative part of the 10 degrees model of Rayleigh-Bénard convection presented in [16] and (ii) to determine the algebraic properties of heating by viscous dissipation. In Section 2 the truncated dynamical system is presented. The conservation laws are derived in Section 3 with a physical interpretation in Section 4. The dynamics is represented in terms of a Nambu system using two conservation laws (Section 5) from which a noncanonical Hamiltonian structure can be derived (Section 6). The viscous heating effect is shown to possess a symplectic as well as a metric structure (Section 7). Results are summarized and discussed in Section 8.

\section{Truncated equations}

The physical setting is the two-dimensional Rayleigh-Bénard convection driven by a vertical temperature contrast $\Delta T$ across the height $H$. Dynamics in the vertical plane $(x, z)$ follows the Boussinesq approximation for incompressible velocity $u=-\partial_{z} \psi, v=\partial_{x} \psi$, with the stream-function $\psi$, and the linearized equation of state $\rho=\rho_{0}(1-\alpha T)$ with the thermal expansion coefficient $\alpha$. Temperature is decomposed according to $T=T_{0}-z \Delta T / H+\theta$ in a mean temperature $T_{0}$, a linear temperature gradient and the perturbation $\theta$. Boundary conditions at $z=0$ and $z=H$ along the $x$-axis demand vanishing vertical velocity, $\psi=0$, vorticity, $\nabla^{2} \psi=0$, and temperature perturbation, $\theta=0$.

The main extension of the Boussinesq equations suggested by [16] is the inclusion of viscous heating in the temperature balance

$$
\begin{array}{r}
\partial_{t} \nabla^{2} \psi+J\left(\psi, \nabla^{2} \psi\right)=g \alpha \partial_{x} \theta+\nu \nabla^{4} \psi \\
\partial_{t} \theta+J(\psi, \theta)=\frac{\Delta T}{H} \partial_{x} \psi+k \nabla^{2} \theta+\frac{\nu}{C_{v}} \partial_{i j} \psi \partial_{i j} \psi
\end{array}
$$

here $\nu$ is viscosity, $k$ heat conductivity, $C_{v}$ is heat capacity, and $g$ is gravity acceleration. Non-dimensional equations are obtained by $x=H x^{\prime}, t=H^{2} t^{\prime} / k$, $\psi=k \psi^{\prime}$ and $\theta=k \nu \theta^{\prime} / g \alpha H^{3}$.

The impact of heating by viscous dissipation in the Fourier decomposition requires at least two modes for the stream-function $\Psi_{n, m}$ and the temperature perturbation $\theta_{n, m}$, furthermore, two modes for the perturbation of the stratification need to be included, $\theta_{0,2}$ and $\theta_{0,4}$ [16]. The Fourier modes are associated with the wave vectors $(\pi a m, \pi n)$. Shear flow is excluded here by $\Psi_{0,1}=0$. Real and imaginary parts of the stream-function are split according to $\Psi_{1,1}=\alpha\left(X_{1}+i X_{2}\right), \Psi_{2,2}=\alpha\left(A_{1}+i A_{2}\right)$, and the temperature perturbation is $\theta_{1,1}=\beta\left(Y_{1}+i Y_{2}\right), \theta_{2,2}=\beta\left(B_{1}+i B_{2}\right), \theta_{0,2}=i \gamma Z_{1}$, and $\theta_{0,4}=i \gamma Z_{2}$.

Heating by viscosity is proportional to the Eckert number, $E c=$ $k^{2} /\left(C_{v} \Delta T H^{2}\right)$ and leads to new nonlinear terms in the temperature equations of the Lorenz system. The present analysis concentrates on the conservative parts of the dynamical equations with zero divergence in phase space. The conservative parts are obtained by an operator splitting through the neglect of the diagonal linear forcing terms (see the general decomposition (4) in [12]). 
The conservative parts of the truncated equations retain all nonlinearities

$$
\begin{aligned}
\dot{X}_{1} & =\sigma Y_{2} \\
\dot{X}_{2} & =-\sigma Y_{1} \\
\dot{Y}_{1} & =X_{2} Z_{1}-r X_{2}+5 /(\sqrt{2} \pi) \sigma E c\left(X_{1} A_{1}+X_{2} A_{2}\right) \\
\dot{Y}_{2} & =-X_{1} Z_{1}+r X_{1}+5 /(\sqrt{2} \pi) \sigma E c\left(X_{1} A_{2}-X_{2} A_{1}\right) \\
\dot{A}_{1} & =\sigma / 2 B_{2} \\
\dot{A}_{2} & =-\sigma / 2 B_{1} \\
\dot{B}_{1} & =4 A_{2} Z_{2}-2 r A_{2}-1 /(2 \sqrt{2} \pi) \sigma E c\left(X_{1}^{2}-X_{2}^{2}\right) \\
\dot{B}_{2} & =-4 A_{1} Z_{2}+2 r A_{1}-1 /(\sqrt{2} \pi) \sigma E c X_{1} X_{2} \\
\dot{Z}_{1} & =X_{1} Y_{2}-X_{2} Y_{1} \\
\dot{Z}_{2} & =4 A_{1} B_{2}-4 A_{2} B_{1}
\end{aligned}
$$

The non-conservative terms not considered here can be represented as a linear gradient, $-\nabla_{x} \Phi$, of the potential

$$
\Phi=\sigma\left(X_{1}^{2}+X_{2}^{2}+4 A_{1}^{2}+4 A_{2}^{2}\right)+Y_{1}^{2}+Y_{2}^{2}+B_{1}^{2}+B_{2}^{2}+b Z_{1}^{2}+4 b Z_{2}^{2}
$$

with respect to the 10 variables (3) - (12), see [22]. The notation follows the standard Lorenz-63 model with the Prandtl number $\sigma=\nu / k$ and the relative Rayleigh number $r=R / R_{c}$, with $R=g \alpha H^{3} \Delta T / k \nu, R_{c}=27 \pi^{4} / 4$, and a geometric factor $b$; for further details see $[30,16]$. The symmetries and the instability of these equations have been intensively studied in [16].

\section{Conservation laws}

Due to the absence of linear forcing and dissipative terms in (3) - (12) the phase space divergence vanishes and the equations satisfy the Liouville theorem. The equations have several time independent conservation laws (determined by the Reduce package CONLAW1 [35]).

The first conservation law which is preserved for arbitrary Eckert number $E c$ represents the sum of kinetic and potential energy and is denoted as Hamiltonian

$$
H=\frac{1}{2}\left(X_{1}^{2}+X_{2}^{2}+4 A_{1}^{2}+4 A_{2}^{2}-2 \sigma Z_{1}-\sigma Z_{2}\right)
$$

Further conservation laws are found for $E c=0$ : The first is quadratic in the temperature perturbations

$$
C=\frac{1}{2}\left(Y_{1}^{2}+Y_{2}^{2}+B_{1}^{2}+B_{2}^{2}+Z_{1}^{2}+Z_{2}^{2}-2 r Z_{1}-r Z_{2}\right)
$$

and the second is based on a coupling between stream-function and temperature perturbation

$$
\hat{C}=\frac{1}{2}\left(X_{1} Y_{1}+X_{2} Y_{2}+A_{1} B_{1}+A_{2} B_{2}\right)
$$


The conserved quantities $C$ and $\hat{C}$ are denoted as Casimir functions according to there specific interpretation in Hamiltonian dynamics (see Section 6).

The Hamiltonians and the Casimirs for the two wave numbers considered are conserved individually (pairs of variables $X, Y$ for $(n, m)=(1,1)$, and $(A, B)$ for $(2,2))$. The sum of $H$ and $C$ has been analysed in a related truncation [6, 34] (denoted as " $Q$ ").

The time derivative of the Casimir $C$ is linear in $E c$

$$
\begin{aligned}
\frac{d C}{d t} & =\frac{\sigma E c}{2 \sqrt{2} \pi}\left[10 Y_{1}\left(A_{1} X_{1}+A_{2} X_{2}\right)\right. \\
& \left.+10 Y_{2}\left(A_{2} X_{1}-A_{1} X_{2}\right)-B_{1}\left(X_{1}^{2}-X_{2}^{2}\right)-2 B_{2} X_{1} X_{2}\right]
\end{aligned}
$$

The time derivative of the second Casimir $\hat{C}$ is determined by the $E c$-dependent dynamics of $B_{1}$ and $B_{2}$ only

$$
\frac{d \hat{C}}{d t}=\frac{9 \sigma E c}{2 \sqrt{2} \pi}\left(A_{1}\left(X_{1}^{2}-X_{2}^{2}\right)+2 A_{2} X_{1} X_{2}\right)
$$

The time derivative of $C$ in (17) will be used to formulate the $E c$-dependent parts in terms of a gradient system (see the forthcoming Section 7.2).

\section{Physical interpretation of the conservation laws}

The total energy is the sum kinetic and potential energy

$$
H=K E+P E
$$

which are given as integrals in the vertical plane

$$
\begin{aligned}
& K E=\frac{1}{2} \int(\nabla \psi)^{2} \mathrm{~d} V \\
& P E=-\alpha g \int z \theta \mathrm{d} V
\end{aligned}
$$

(see for example [5]).

In the present truncation the kinetic energy $K E$ is given by

$$
K E=\frac{1}{2}\left(X_{1}^{2}+X_{2}^{2}+4 A_{1}^{2}+4 A_{2}^{2}\right)
$$

and total potential energy is

$$
P E=-\frac{\sigma}{2}\left(2 Z_{1}+Z_{2}\right)
$$

The available potential energy characterizes the amount of potential energy which can be converted to kinetic energy $[11,30]$

$$
A P E=-\frac{\alpha g H}{2 \Delta T} \int \theta^{2} \mathrm{~d} V
$$


The truncated version is

$$
A P E=-\frac{\sigma}{2 r}\left(Y_{1}^{2}+Y_{2}^{2}+B_{1}^{2}+B_{2}^{2}+Z_{1}^{2}+Z_{2}^{2}\right)
$$

Note that $A P E \leq 0$ since any perturbation reduces the energy transformable to kinetic energy. Total energy and the Casimir $C$ are related to $K E$ and $A P E$ by

$$
H=K E+A P E+\frac{\sigma}{r} C
$$

Therefore, the Casimir contribution can be identified as unavailable potential energy [5]

$$
\frac{\sigma}{r} C=P E-A P E
$$

The balance between kinetic and available potential energy is given by the Lorenz energy cycle

$$
\begin{gathered}
\frac{d}{d t} K E=\sigma\left(X_{1} Y_{2}-X_{2} Y_{1}+2 A_{1} B_{2}-2 A_{2} B_{1}\right) \\
\frac{d}{d t} A P E=-\frac{d}{d t} K E-\frac{\sigma}{r} \frac{d C}{d t}
\end{gathered}
$$

For finite Eckert number $(E c>0)$ viscous heating modifies available potential energy (see $d C / d t$ in (17)). The balance between $K E$ and $A P E$ is closed, $d K E / d t+d A P E / d t=0$, in the absence of viscous heating. The sum $E$ of kinetic and available potential energy is the available energy

$$
E=K E+A P E
$$

and will be used represent the viscous heating term (see (38) below).

The Casimir functions $(15,16)$ correspond to the

Continuous versions of the Casimir functions are $[2,27] C_{1}=\int g(T-z) \mathrm{d} V$ and $C_{2}=\int \zeta h(T-z) \mathrm{d} V$, where $T$ is the scaled deviation of the temperature from a linear profile $z, \zeta$ is vorticity; the integrals are in the vertical plane. $C_{1}$ is based on the preservation of $T-z$ contours and corresponds to $C$ in (15) for the function $g(x)=x^{2} / 2 . C_{2}$ depends on Kelvin's circulation theorem and corresponds to $\hat{C}$ in (16). In the continuos Nambu representation of [2] the Casimir $C_{2}$ is used together with the Hamiltonian $H=\int\left(1 / 2(\nabla \psi)^{2}-T z\right) \mathrm{d} V$, while [27] compare different Nambu representations using $H, C_{1}$ (for $h(x)=x$ ), enstrophy and total buoyancy $\left(C_{1}\right.$ with $\left.g(x)=x\right)$. In the present analysis the first Casimir $C$ in (15), i.e. the unavailable potential energy is used in order to continue previous work on the Nambu representation of the Lorenz-63 model [22]. A further motivation for using this Casimir is its role in the viscous heating (see Section 7.2). 


\section{Nambu structure}

Névir and Blender [22] developed a Nambu representation of the nondissipative parts of the classical Lorenz-63 [12] model for $X_{1}, Y_{2}$, and $Z_{1}$ (see also $[1,24,3]$ and [25] for a classification). With the conservation laws for $H$ and $C$ the Lorenz equations are in terms of $x=\left(X_{1}, Y_{2}, Z_{1}\right)$

$$
\frac{d}{d t} x_{i}=-\varepsilon_{i j k} C_{j} H_{k}, \quad i, j, k=1,2,3
$$

with the Levi-Civita symbol $\varepsilon_{i j k}$ (note that the definitions in [22] are interchanged). The second Casimir $\hat{C}$ (16) does not occur in the classical Lorenz-63 truncation since $Y_{1}$ and $X_{2}$ are not considered.

Rewriting the dynamical system (3) - (12) for the 10-component variable vector

$$
x=\left(X_{1}, Y_{2}, Z_{1}, X_{2}, Y_{1}, A_{1}, B_{2}, Z_{2}, A_{2}, B_{1}\right)
$$

the Nambu equations for the extended Lorenz system is

$$
\frac{d x}{d t}=\mathcal{N}\left(\nabla_{x} C, \nabla_{x} H\right)
$$

with the Nambu tensor $\mathcal{N}$ and the gradient $\nabla_{x}$.

The non-vanishing elements of the Nambu tensor are

$$
N_{1,2,3}=-\varepsilon_{123}, \quad N_{3,4,5}=\varepsilon_{345}, \quad N_{6,7,8}=-\varepsilon_{678}, \quad N_{8,9,10}=\varepsilon_{8910}
$$

with the standard definition of the Levi Civita symbol $\varepsilon$. Thus the Nambu tensor is cyclic and anti-symmetric. Furthermore, it satisfies the generalized Jacobi identity [33, 31]

$$
N_{i j k} N_{l p q}+N_{i j q} N_{l k p}+N_{i j p} N_{l q k}=0
$$

in the form required for constant Nambu tensors.

Based on (33) a Nambu bracket for arbitrary phase space functions $F(x)$ is written

$$
\frac{d F}{d t}=\{F, C, H\}
$$

which is cyclic $\{F, C, H\}=\{C, H, F\}=\{H, F, C\}$. This cyclicity is the main ingredient in applications of the Nambu representation in numerical models [28]. This bracket vanishes for the second Casimir (16), $\{\hat{C}, C, H\}=0$.

The Nambu representation of the conservative terms can be presented in 
terms of brackets

$$
\frac{d}{d t}\left(\begin{array}{c}
X_{1} \\
Y_{2} \\
Z_{1} \\
X_{2} \\
Y_{1} \\
A_{1} \\
B_{2} \\
Z_{2} \\
A_{2} \\
B_{1}
\end{array}\right)=\left(\begin{array}{rrrr}
\left\{Z_{1}, Y_{2}\right\} & & \\
\left\{X_{1}, Z_{1}\right\} & & \\
\left\{Y_{2}, X_{1}\right\} & +\left\{X_{2}, Y_{1}\right\} & \\
& \left\{Y_{1}, Z_{1}\right\} & \\
& \left\{Z_{1}, X_{2}\right\} & \\
& & \left\{Z_{2}, B_{2}\right\} & \\
& & \left\{A_{1}, Z_{2}\right\} & \\
& & \left\{B_{2}, A_{1}\right\} & +\left\{A_{2}, B_{1}\right\} \\
& & \left\{B_{1}, Z_{2}\right\} \\
& & & \left\{Z_{2}, A_{2}\right\}
\end{array}\right)
$$

where the bracket $\{$,$\} is the Jacobian of C$ and $H$ with respect to $a$ and $b$, $\{a, b\}=\partial C / \partial a-\partial H / \partial b$. The three brackets in the upper left represent the Nambu dynamics of the Lorenz-63 (31) model [22]. This structure demonstrates the decoupling of the two wave number modes $(m, n)=(1,1)$ and $(2,2)$ in the five upper and the five lower rows. Furthermore, the coupled Fourier modes are separated in the columns. Coupling between the wave number modes is given by the feedback represented in the Eckert terms which is not included here (see Section 7).

Instead of the Hamiltonian the dynamical equations can be rewritten using the available energy $E=K E+A P E$ in the Nambu bracket (36)

$$
\frac{d F}{d t}=\{F, C, E\}
$$

since $E$ deviates from $H$ only by the Casimir

$$
H=E+\frac{\sigma}{r} C
$$

which is annihilated in the bracket.

\section{Hamiltonian structure}

The Hamiltonian structure of the dynamical system (3) - (12) is a straightforward evaluation of the Nambu system (33) (also for $E c=0$ )

$$
\frac{d}{d t} x=\mathcal{P} \nabla_{x} H=\mathcal{N}\left(\nabla_{x} C, \nabla_{x} H\right)
$$

with the anti-symmetric Poisson tensor

$$
\mathcal{P}(.)=\mathcal{N}\left(\nabla_{x} C, .\right)
$$


The elements of the Poisson tensor are explicitly given by derivatives of the Casimir with respect to the variables in (32), for example $C_{1}=\partial C / \partial X_{1}$, etc.

$$
\mathcal{P}=\left(\begin{array}{cccccccccc}
0 & C_{3} & -C_{2} & 0 & 0 & 0 & 0 & 0 & 0 & 0 \\
-C_{3} & 0 & 0 & 0 & 0 & 0 & 0 & 0 & 0 & 0 \\
C_{2} & 0 & 0 & -C_{5} & 0 & 0 & 0 & 0 & 0 & 0 \\
0 & 0 & C_{5} & 0 & -C_{3} & 0 & 0 & 0 & 0 & 0 \\
0 & 0 & 0 & C_{3} & 0 & 0 & 0 & 0 & 0 & 0 \\
0 & 0 & 0 & 0 & 0 & 0 & C_{8} & -C_{7} & 0 & 0 \\
0 & 0 & 0 & 0 & 0 & -C_{8} & 0 & 0 & 0 & 0 \\
0 & 0 & 0 & 0 & 0 & C_{7} & 0 & 0 & -C_{10} & 0 \\
0 & 0 & 0 & 0 & 0 & 0 & 0 & C_{10} & 0 & -C_{8} \\
0 & 0 & 0 & 0 & 0 & 0 & 0 & 0 & C_{8} & 0
\end{array}\right)
$$

The Poisson tensor $\mathcal{P}$ defines a Poisson bracket for the dynamics of arbitrary phase space functions $F(x)$

$$
\frac{d F}{d t}=\{F, H\}_{P}
$$

The Casimir $C$ is conserved as the nullspace of the Poisson tensor

$$
\mathcal{P} \nabla_{x} C=0
$$

Thus, in Hamiltonian dynamics the conservation of Casimirs is an inherent property hidden in the Poisson tensor. These conservation laws lead to foliations in phase space and are interpreted as a kinematic property of the dynamical system, whereas the conservation of the Hamiltonian is considered as a dynamic property.

\section{Viscous heating by Eckert terms}

An important property of the $E c$-dependent terms in the equations for $Y_{1}, Y_{2}, B_{1}$, and $B_{2}(5),(6)$, (9), and (10) is that they satisfy the Liouville theorem. Thus a representations in terms of a stream-function is possible (hence as Hamiltonian systems). To obtain this we consider the equations for $Y_{1}, Y_{2}$ and $B_{1}, B_{2}$ in two complex plains given by $Y=Y_{1}+i Y_{2}$ and $B=B_{1}+i B_{2}$. The dynamic equations are determined by the gradient of the complex function

$$
f(Y, B)=\frac{\sigma E c}{2 \sqrt{2} \pi}\left(10 X^{*} A Y^{*}-X X B^{*}\right)
$$

with $X=X_{1}+i X_{2}$ and $A=A_{1}+i A_{2}$. The Cauchy-Riemann equations yield the dynamics for $Y$ and $B$ in terms of a stream-function $(\operatorname{Im} f)$ and the gradient of potential $(\operatorname{Re} f)$. It turns out that the potential is given by the time derivative of the Casimir $C$ (unavailable potential energy) which leads to a metric system [19]. The stream-function is considered as a second Hamiltonian $\tilde{H}$ (not to be confused with (14)), thus the complex function is $f=\dot{C}+i \tilde{H}$. In the following the Hamiltonian and the gradient representation are considered. 


\subsection{Hamiltonian system}

The Hamiltonian $\tilde{H}=\operatorname{Im} f(45)$ can be constructed by the Ec-dependent forcing terms for the set of variables $y_{j}=\left\{Y_{2}, Y_{1}, B_{2}, B_{1}\right\}$ in (32)

$$
\frac{d}{d t} y_{j}=G\left[y_{j}\right]
$$

using that these terms $G$ depend only on $X_{1}, X_{2}, A_{1}, A_{2}$. Therefore, $H$ is formally

$$
\tilde{H}=Y_{2} G\left[Y_{1}\right]-Y_{1} G\left[Y_{2}\right]+B_{2} G\left[B_{1}\right]-B_{1} G\left[B_{2}\right]
$$

which can be rearranged in the compact representation

$$
\tilde{H}=Y_{2}^{2} \frac{d}{d t} \frac{Y_{1}}{Y_{2}}+B_{2}^{2} \frac{d}{d t} \frac{B_{1}}{B_{2}}
$$

determined by the phases of $\theta_{1,1}=\beta\left(Y_{1}+i Y_{2}\right)$ and $\theta_{2,2}=\beta\left(B_{1}+i B_{2}\right)$. In terms of the dynamical variables, $\tilde{H}$ has the explicit form

$$
\begin{aligned}
\tilde{H} & =\frac{\sigma E c}{2 \sqrt{2} \pi}\left[10 Y_{2}\left(A_{1} X_{1}+A_{2} X_{2}\right)\right. \\
& \left.-10 Y_{1}\left(A_{2} X_{1}-A_{1} X_{2}\right)-B_{2}\left(X_{1}^{2}-X_{2}^{2}\right)+2 B_{1} X_{1} X_{2}\right]
\end{aligned}
$$

Thus the viscous heating effect can be represented by a second Hamiltonian part

$$
\frac{d}{d t} y=\mathcal{P}_{E c} \nabla_{y} \tilde{H}
$$

where $\nabla_{y}$ is the gradient with respect to $y$. The Poisson tensor is canonical with the non-zero elements

$$
\begin{array}{rll}
\mathcal{P}_{E c}\left(Y_{1}, Y_{2}\right) & =-1, & \mathcal{P}_{E c}\left(Y_{2}, Y_{1}\right)=1 \\
\mathcal{P}_{E c}\left(B_{1}, B_{2}\right) & =-1, & \mathcal{P}_{E c}\left(B_{2}, B_{1}\right)=1
\end{array}
$$

The dynamics (50) has a canonical Poisson bracket

$$
\{F, \tilde{H}\}_{E c}=\nabla_{x} F \mathcal{P}_{E c} \nabla_{x} \tilde{H}
$$

which conserves the Hamiltonian (14), $\{H, \tilde{H}\}_{E c}=0$. Thus $H$ can formally be considered as a Casimir of the tensor $\mathcal{P}_{E c}, \mathcal{P}_{E c} H=0$.

\subsection{Gradient System}

The Ec-dependent terms in (3) - (12) are the gradient of a potential given by the real part $\operatorname{Re} f(45)$. This potential can be identified as time derivative of the Casimir (17). Hence the present low order system shows a relationship between the Hamiltonian part (42) and the viscous heating mediated by the Casimir (15). The potential can be constructed from the Ec-dependent forcing terms (46) according to

$$
\frac{d C}{d t}=Y_{1} G\left[Y_{1}\right]+Y_{2} G\left[Y_{2}\right]+B_{1} G\left[B_{1}\right]+B_{2} G\left[B_{2}\right]
$$


Embedding this in the dynamics of $x$ the gradient representation is

$$
\frac{d}{d t} x=\mathcal{M} \nabla_{x} \frac{d C}{d t}
$$

with the symmetric operator $\mathcal{M}=\operatorname{diag}(0,1,0,0,1,0,1,0,0,1)$ which selects the variables $Y_{2}, Y_{1}, B_{1}, B_{2}$ in the complete 10-component vector $x$ (32). Thus for an arbitrary phase space function $F$ the contribution of viscous heating (54) can be included in a symmetric bracket (also denoted as metric bracket in contrast to symplectic Poisson brackets).

$$
\left\langle F, \frac{d C}{d t}\right\rangle=\nabla_{x} F \mathcal{M} \nabla_{x} \frac{d C}{d t}
$$

Due to the Cauchy-Riemmann equations the $\tilde{H}$ manifolds are perpendicular to $\dot{C}$ and $\tilde{H}$ is preserved in this bracket, $\langle\tilde{H}, \dot{C}\rangle=0$. With (38) the dynamics of the full system can be completely understood in terms of the available energy E

$$
\frac{d F}{d t}=\{F, C, E\}-\frac{r}{\sigma}\left\langle F, \frac{d E}{d t}\right\rangle
$$

since

$$
\frac{d C}{d t}=-\frac{r}{\sigma} \frac{d E}{d t}
$$

represents the change in available energy.

This symmetric bracket does not represent physical dissipation associated with the convergence of trajectories in phase space. The reason is that the dissipated energy is re-introduced in the temperature equation which is represented by dynamical degrees of freedom.

A main property of this symmetric bracket is that it generates a positive change of the Casimir tendency

$$
\langle\dot{C}, \dot{C}\rangle=\frac{\sigma^{2} E c^{2}}{8 \pi^{2}}\left(100\left(A_{1}^{2}+A_{2}^{2}\right)\left(X_{1}^{2}+x_{2}^{2}\right)+\left(X_{1}^{2}+X_{2}^{2}\right)^{2}\right) \geq 0
$$

note that this is proportional to $E c^{2}$. Since the Nambu bracket representing the conservative part does not vanish $\{\dot{C}, C, E\} \neq 0$, the time derivative of the Casimir tendency in the full equations has no definite sign

$$
\frac{d}{d t} \dot{C}=\{\dot{C}, C, E\}+\langle\dot{C}, \dot{C}\rangle
$$

Due to this property, $\dot{C}$ cannot be considered as the analogue to entropy (see the concept in [9]).

Since the Hamiltonian is conserved for arbitrary Eckert number the symmetric bracket vanishes for $H$

$$
\frac{d H}{d t}=\langle H, \dot{C}\rangle=0
$$

Hence the Hamiltonian can be considered as a nullvector of the symmetric bracket [9] (see the construction method [19]). 
The role of the Casimir is twofold: (i) As a conserved quantity it determines the non-viscous dynamics in the Nambu representation, and (ii) the time derivative determines the heating effect in a gradient system.

\section{Summary and Conclusions}

This paper discusses the impact of viscous heating on the dynamic properties of convection. The model is given by a truncation of the Boussinesq equations [30] with an additional heating caused by viscous dissipation [16]. Wave numbers selected are $n=m=1$ and $n=m=2$ for the stream function and the temperature perturbation, where additional vertical wave numbers $m=2$ and $m=4$ are included. Vertical shear is not incorporated. Linear forcing and friction are removed in the dynamical equations to obtain a divergence free dynamical system satisfying Liouville's theorem.

The truncated systen shows one conservation law for all $E c$ which is given by the sum of kinetic and potential energy and identified as the Hamiltonian of the dynamical system. There are two quadratic conservation laws for vanishing Eckert number, $E c=0$, a first, $C$, related to unavailable potential energy (UPE, the residuum between total energy and available potential energy) and a second, $\hat{C}$, related to the coupling of stream-function and temperature perturbations. Both conservation laws are Casimirs of the Hamiltonian representation. The viscous heating changes both Casimir functions. An available potential energy APE is identified in the truncated system which is convertible to kinetic energy. The Lorenz energy cycle for kinetic and available potential energy has a source term linear in $E c$.

A Nambu representation is defined using the Hamiltonian $H$ and the Casimir $C$ to determine the time derivative of an arbitrary state space function by a Nambu bracket, $\dot{F}=\{F, C, H\}$. The Nambu bracket is cyclic and satisfies a generalized Jacobi identity. In a Nambu representation the Casimirs can be considered as additional Hamiltonians. The Nambu representation can be directly transformed into noncanonical Hamiltonian dynamics by evaluating the Casimir dependency in the Nambu bracket, $\{F, C, H\}=\{F, H\}_{P}$.

Casimirs and the Hamiltonian are excellent observables for the study of the response of dynamical system to small perturbations far from equilibrium $[26,13,14]$. Even when these quantities are not conserved in the full nonlinear equations, they provide an intrinsic skeleton characterizing the geometry of the phase space trajectories. Promising results have been obtained recently for perturbations of the Lorenz 96 model considering total energy and momentum analogues [18].

The final result of the present publication is that the dynamics (3) - (12) can be decomposed in a Nambu representation and a metric representation of the viscous heating which is proportional to $E c$

$$
\dot{F}=\{F, C, H\}+\langle F, \dot{C}\rangle
$$

The Casimir $C$ appears in the Nambu as well as in the metric term (via the time derivative). Furthermore, the viscous heating bracket is equivalent to a 
canonical Hamiltonian bracket, $\langle F, \dot{C}\rangle=\{F, \tilde{H}\}_{E c}$, due to the Cauchy-Riemann equations.

The present low order model revels specific properties of the viscous heating which is usually neglected in models:

1. Viscous heating does not perturb conservation of total energy (the Hamiltonian). This result may have implications for considering this effect in complex models.

2. The viscous heating terms are divergence free in phase space, hence the full dynamical system satisfies the Liouville theorem.

3. The Lorenz energy cycle for kinetic and available energy has a source term given by viscous heating. This effect alters convective processes by modifying available potential energy.

4. Viscous heating determines the time derivative of the Casimir in the Nambu representation. Nevertheless, its role in the Nambu and the Hamiltonian representation is unaltered.

5. The viscous heating terms can be represented by a symmetric bracket with a positive change of the Casimir time derivative, $\langle\dot{C}, \dot{C}\rangle \geq 0$. However, the interpretation of $\dot{C}$ as an entropy according to [9] is not possible since the Poisson bracket (43) does not vanish, $\{\dot{C}, H\}_{P} \neq 0$.

This study shows that a correct energy recycling budget leads to conservation of total energy and retains the Hamiltonian and Nambu structures. The single impact is a change of the Casimir function, without altering it's role in the Nambu representation (such conservation laws have been termed constitutive in Nambu dynamics $[23,27]$ ). The role of the Casimir in the recycling process might be a particularity of the present model, but the feedback in the Lorenz energy cycle and the recreation of kinetic energy could shed new light on the relationships with entropy $[9,19]$. The Ec-term mixes available potential energy (APE) and unavailable potential energy. This implies that an improper treatment of the Ec-term can have a large impact on the energy cycle [11,17] and bias considerably the thermodynamic efficiency of a geophysical fluid [17, 7, 8, 15].

[1] M. Axenides and E. Floratos. Strange attractors in dissipative Nambu mechanics: classical and quantum aspects. J. of High Energy Phys., 2010:133, 2010. 10.1007/JHEP04(2010)036.

[2] A. Bihlo. Rayleigh-Bénard convection as a Nambu-metriplectic problem. J. Phys. A, 41:292001, 2008.

[3] A. Bihlo and J. Staufer. Minimal atmospheric finite-mode models preserving symmetry and generalized hamiltonian structures. Physica D: Nonlinear Phenomena, 240(7):599 - 606, 2011.

[4] A. Gluhovsky. Energy-conserving and Hamiltonian low-order models in geophysical fluid dynamics. Nonlin. Proc. Geophys., 13:125-133, 2006.

[5] A. Gluhovsky and C. Tong. The structure of energy conserving low-order models. Phys. Fluids, 11:334-342, 1999. 
[6] L. N. Howard and R. Krishnamurti. Large-scale flow in turbulent convection: a mathematical model. J. Fluid Mech., 170:385-410, 1986.

[7] D. R. Johnson. Adv. Geophys., 31:43, 1989.

[8] D. R. Johnson. Entropy, the Lorenz energy cycle, and climate. In D. A. Randall, editor, General Circulation Model Development: Past, Present and Future, pages 659-720. Academic Press, New York, 2000.

[9] A. N. Kaufman. Dissipative Hamiltonian systems: A unifying principle. Phys. Lett. A, 100:419-422, 1984.

[10] Y. Kuroda. On the Casimir invariant of Hamiltonian fluid mechanics. J. Phys. Soc. Japan, 60:727-730, 1991.

[11] E. N. Lorenz. Available potential energy and the maintenance of the general circulation. Tellus, 7:157167, 1955.

[12] E. N. Lorenz. Deterministic nonperiodic flow. J. Atmos. Sci., 20:130, 1963.

[13] V. Lucarini. Response theory for equilibrium and non-equilibrium statistical mechanics: Causality and generalized Kramers-Kronig relations. J. Stat. Phys., 131:543558, 2008.

[14] V. Lucarini. Evidence of dispersion relations for the nonlinear response of the Lorenz 63 system. J. Stat. Phys., 134:381400, 2009.

[15] V. Lucarini. Thermodynamic efficiency and entropy production in the climate system. Phys. Rev. E, 80:021118, 2009.

[16] V. Lucarini and K. Fraedrich. Symmetry breaking, mixing, instability, and low-frequency variability in a minimal Lorenz-like system. Phys. Rev. E, 80:026313, 2009.

[17] V. Lucarini and F. Ragone. Energetics of climate models: Net energy balance and meridional enthalpy transport. Rev. Geophys., 49:RG1001, 2011.

[18] V. Lucarini and S. Sarno. A statistical mechanical approach for the computation of the climatic response to general forcings. Nonlin. Processes Geophys., 18:728, 2011.

[19] P. J. Morrison. A paradigm for joinded Hamiltonian and dissipative systems. Physica D, 18:410-419, 1986.

[20] Y. Nambu. Generealized Hamiltonian dynamics. Phys. Rev. D, 7:2403, 1973.

[21] P. Névir and R. Blender. A Nambu representation of incompressible hydrodynamics using helicity and enstrophy. J. Phys. A, 26:L1189-1193, 1993. 
[22] P. Névir and R. Blender. Hamiltonian and Nambu representation of the non-dissipative Lorenz equations. Beitr. Phys. Atmosph., 67:133, 1994.

[23] P. Névir and M. Sommer. Energy-vorticity theory of ideal fluid mechanics. J. Atmos. Sci., 66:2037-2084, 2009.

[24] V. Pelino and F. Maimone. Energy cycle for the Lorenz attractor. preprint.

[25] Z. Roupas. Phase space geometry and chaotic attractors in the dissipative decomposition. preprint arXiv:1110.0766v2.

[26] D. Ruelle. General linear response formula in statistical mechanics, and the fluctuation-dissipation theorem far from equilibrium. Phys. Lett. A, 245:220-224, 1998.

[27] R. Salazar and M. V. Kurgansky. Nambu brackets in fluid mechanics and magnetohydrodynamics. J. Phys. A, 43:305501(1-8), 2010.

[28] R. Salmon. A general method for conserving quantities related to potential vorticity in numerical models. Nonlinearity, 18:R1-16, 2005.

[29] R. Salmon. A general method for conserving energy and potential enstrophy in shallow water models. J. Atmos. Sci., 64:515-531, 2007.

[30] B. Saltzman. Finite amplitude free convection as an initial value problem - I. J. Atmos. Sci., 19:329-341, 1962.

[31] M. Sommer, K. Brazda, and M. Hantel. Algebraic construction of a Nambu bracket for the two-dimensional vorticity equation. Phys. Lett. A, 375:3310$3313,2011$.

[32] M. Sommer and P. Névir. A conservative scheme for the shallow-water system on a staggered geodesic grid based on a Nambu representation. Quart. J. Roy. Meteorol. Soc., 135:485-494, 2009.

[33] L. Takhtajan. On foundation of the generalized Nambu mechanics. Commun. Math. Phys., 160:295-315, 1994.

[34] J. L Thiffeault and W. Horton. Energy-conserving truncations for convection with shear flow. Phys. Fluids, 8:1715-1719, 1996.

[35] T. Wolf. Manual for CONLAW versions 1,2,3,4. REDUCE Packages. 